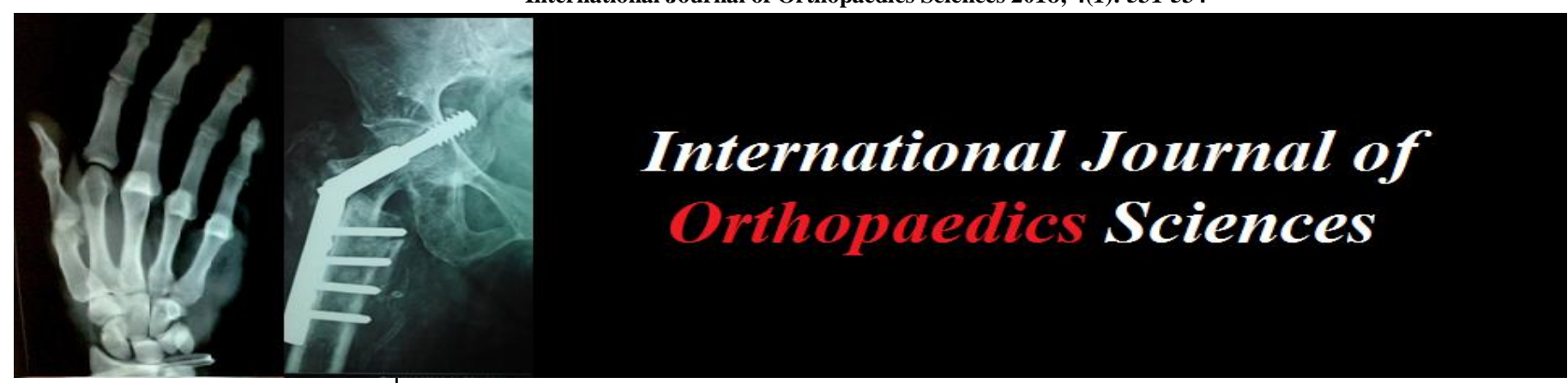

ISSN: $2395-1958$

IJOS 2018; 4(1): 531-534

(C) 2018 IJOS

www.orthopaper.com

Received: 27-11-2017

Accepted: 28-12-2017

Prashant Acharya

Fr. Muller Hospital, Mangalore, Karnataka, India

Arjun Naik

Fr. Muller Hospital, Mangalore, Karnataka, India

\section{Adding dynamic stability to lateral ligament reconstruction in ankle injuries}

\section{Prashant Acharya and Arjun Naik}

DOI: https://doi.org/10.22271/ortho.2018.v4.i1h.77

\begin{abstract}
Introduction: Ankle sprains involving the anterior talo-fibular ligament are one of the commonest injuries encountered in the day to day orthopaedic practice. Routinely these chronic instabilities are also initially treated non-operatively. Surgical intervention is required if the conservative treatment fails or in individuals demanding rapid return to athletic activities. The commonly performed surgeries for these injuries involve reconstruction of Anterior Talo-fibular ligament. We are describing a non anatomic technique using Semitendinosus graft for the reconstruction of lateral structures, including anterior talofibular and calcaneo-fibular ligament.

Materials and Methods: A total of 6 patients who sustained trauma to their ankle more than 6 months prior to presentation in the form of an inversion injury came with complaints of pain and instability in the respective ankles. There was an increase in the lateral clear space with lateral tilt of talus on the radiograph. MRI scan showed loss of continuity of anterior talofibular ligament and bone marrow oedema in the dome of talus and anterior aspect of lower tibia. All patients were treated surgically using our technique.

Results: The mean FAOS significantly improved from 33.63 points to 81.96 and VAS showed a decrease from 7.8 to 4.3 in 6 months.

Conclusion: Our modification can enhance the lateral stability as two strands of the tendon control the inversion laxity. In this way our technique gives extra importance to the calcaneo-fibular ligament without reducing the strength of peroneus brevis muscle. At the same time we also ensure dynamic stability of the ankle by suturing the ATFL arm of the graft to peroneus brevis so that we recreate the normal gait mechanics. We found this study to give excellent results in subtalar instability and tibio-talar instability without any donor site morbidity.
\end{abstract}

Keywords: Ankle instability, Lateral ligament reconstruction, ankle dynamic stability

\section{Introduction}

Ankle sprains involving the anterior talo-fibular ligament are one of the commonest injuries encountered in the day to day orthopaedic practice. The treatment of these ankle injuries is fairly simple, with surgical intervention rarely required. However in a small subset of patients these 'apparently minor' injuries can progress to chronic instability leading to significant disability. Routinely these chronic instabilities are also initially treated non-operatively. Surgical intervention is required if the conservative treatment fails or in individuals demanding rapid return to athletic activities. The commonly performed surgeries for these injuries involve reconstruction of Anterior Talo-fibular ligament in some form. It can be broadly classified into anatomic repair, anatomic reconstruction and non-anatomic reconstruction ${ }^{[1]}$. We are describing a non anatomic technique using Semitendinosus graft for the reconstruction of lateral structures, including anterior talo-fibular and calcaneo-fibular ligament.

The most common techniques are Bostrom, modified Bostrom and modified Elmslie operation $[2,3]$. Around 50-80 techniques have been defined in the literature for reconstruction of the ligament ${ }^{[4]}$. We have modified the Chrisman-Snook method of reconstruction and found to have favourable outcome.

\section{Materials and Methods}

This study was conducted in Father Muller Medical college hospital. A total of 6 patients who sustained trauma to their ankle more than 6 months prior to presentation in the form of an 
inversion injury came with complaints of pain and instability in the respective ankles. 4 out of 6 patients had undergone an $\mathrm{X}$-ray at the time of initial injury and were treated conservatively for ligament sprain with rest and analgesics.

On follow up the pain had subsided but the instability persisted, especially while walking on uneven surface. 2 out of 6 patients had undergone an intensive rehab program involving evertor strengthening and proprioception exercises but showed no improvement. The remaining 4 patients underwent rehabilitation programme after presenting to us. Despite 4 months of extensive rehabilitation the instability persisted. Clinical evaluation at this stage revealed a positive Inversion stress and anterior Drawer test for ankle. There was an increase in the lateral clear space with lateral tilt of talus on the radiograph. MRI scan showed loss of continuity of anterior talofibular ligament and bone marrow oedema in the dome of talus and anterior aspect of lower tibia. All patients were treated surgically using our technique.

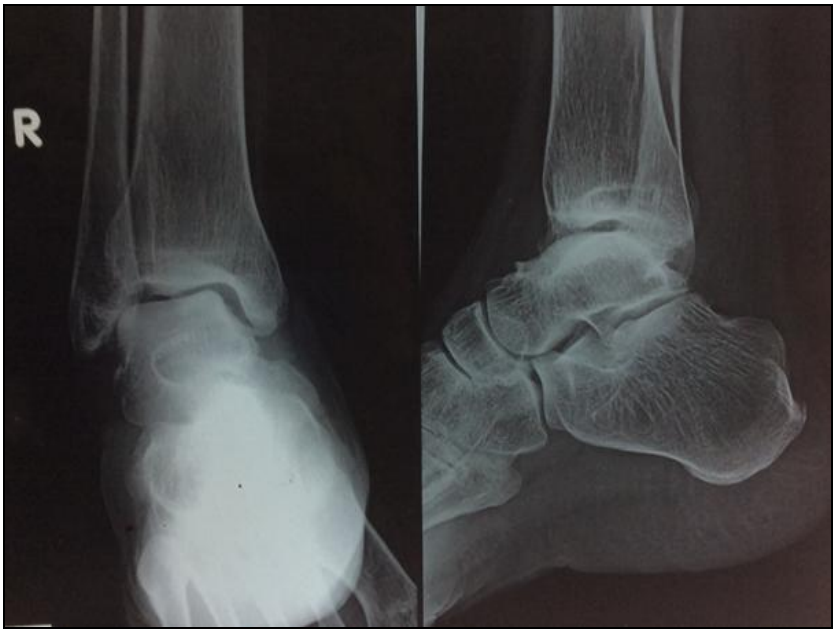

Fig 1: Radiograph showing increased lateral clear space and varus tilting of the talus

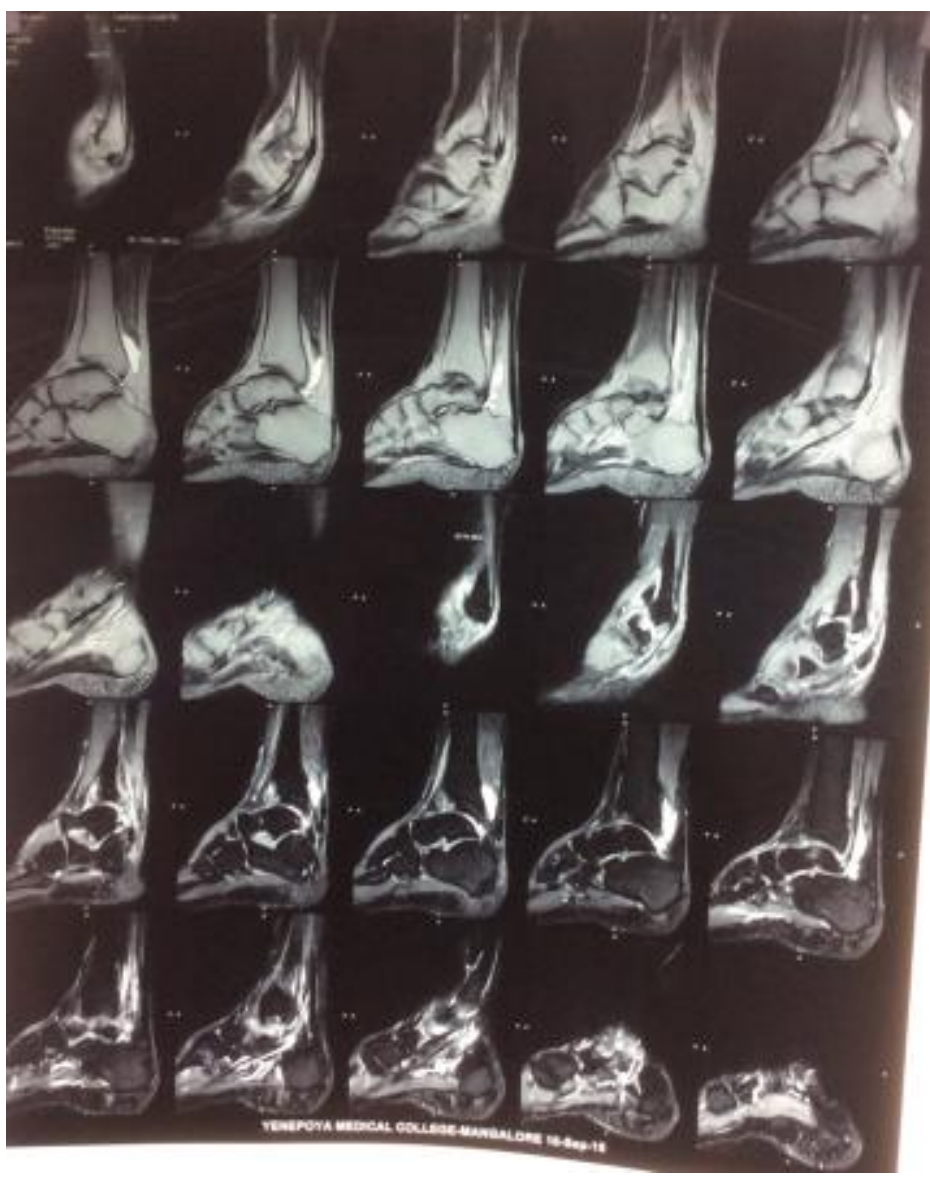

Fig 2: MRI showing a torn ATFL

\section{Operative technique}

Considering the failure of conservative management and the persistent instability it was decided to operate on the patients. Initial diagnostic arthroscopy revealed grade 1 cartilage changes in the non-weight bearing part of talus in 2 patients, no cartilage changes were seen in 4 patients and ankle instability was confirmed in all. Semitendinosus tendon graft was harvested from the ipsilateral limb, prepared and pretensioned in a standard fashion. Using a curved lateral incision along the course of peroneal tendons the lateral aspect of the respective ankle was exposed. No attempt was made at isolating and repairing the Anterior Talo-fibular ligament. Using a $1.2 \mathrm{~mm}$ drill bit two tunnels were drilled; one in the lateral malleolus in an antero-posterior direction and the other one in the lateral wall of the calcaneum in the supero-inferior direction. The edges of the tunnel were smoothened to avoid damaging the tendon during passage. A $5 \mathrm{~mm}$ suture anchor was used to fix one end of the graft to the base of $5^{\text {th }}$ metatarsal. The fixation was augmented by suturing the graft to the Peroneus brevis tendon using Ethibond suture. The graft was passed through the lateral malleolus anterior to posterior and the through the tunnel in the calcaneum. It was then sutured to itself just anterior to the lateral malleolus maintaining tension on the tendon with ankle in minimal eversion. The ankle was immobilised in a plaster cast for the next one and half month. The patient was adviced non weight bearing for further one and half months. Patients underwent rehabilitation for a period of 6 months at which time The Foot and ankle Outcome Score (FAOS) and Visual analogue scale (VAS) were recorded. 


\section{Results}

All patients were compliant to post-op rehabilitation protocol. Follow-up period was 6 months. The mean age of the subjects was 34 years. The mean FAOS significantly improved from
33.63 points to 81.96 and VAS showed a decrease from 7.8 to 4.3 in 6 months. No patients complained of episodes of instability in the next 6 months. No immediate or late complications were seen.

\begin{tabular}{|c|c|c|c|c|c|c|c|c|}
\hline \multirow[b]{3}{*}{ Parameter } & & \multicolumn{4}{|c|}{ change } & \multirow[b]{3}{*}{ t v alue } & \multirow[b]{3}{*}{$\mathrm{p}$} & \\
\hline & & \multirow[b]{2}{*}{ Mean } & \multirow[b]{2}{*}{ Std. Dev iation } & \multicolumn{2}{|c|}{$\begin{array}{l}95 \% \text { Confidence } \\
\text { Interval of the } \\
\text { Diff erence }\end{array}$} & & & \\
\hline & & & & Lower & Upper & & & \\
\hline Activities of Daily & Pre-op - 6 months & -42.500 & 4.037 & -46.737 & -38.263 & 25.79 & .000 & $\mathrm{HS}$ \\
\hline Pain & Pre-op - 6 months & -47.000 & 1.897 & -48.991 & -45.009 & 60.68 & .000 & $\mathrm{HS}$ \\
\hline Quality of Life & Pre-op - 6 months & -69.500 & 2.739 & -72.374 & -66.626 & 62.16 & .000 & $\mathrm{HS}$ \\
\hline Sports and Recreation & Pre-op - 6 months & -57.500 & 6.892 & -64.733 & -50.267 & 20.44 & .000 & $\mathrm{HS}$ \\
\hline Sy mptoms & Pre-op - 6 months & -25.167 & 5.307 & -30.736 & -19.597 & 11.62 & .000 & $\mathrm{HS}$ \\
\hline Visual Analogue score & Pre-op - 6 months & 3.500 & 1.378 & 2.053 & 4.947 & 6.22 & .002 & $\mathrm{HS}$ \\
\hline
\end{tabular}

\section{Discussion}

Ankle reconstruction is more than 100 years old and is commonly performed for sportsmen and athletes ${ }^{[5]}$. The most common mechanism of injury is when the subject misses a step and the ankle goes into plantarflexion and inversion. It can be reconstructed using anatomic (Brostrom) ${ }^{[6]}$ or nonanatomic (Christman-Snook) techniques. The original Christman-Snook technique reconstructs or reinforces the ATFL and CFL by using one half of the peroneus brevis tendon ${ }^{[7]}$.

Chrisman and Snook concluded that in chronic ankle instability, both talotibial and subtalar joints ligaments have to be reconstructed especially in patients who wish to engage in heavy work or athletics ${ }^{[8]}$. A chronic ligamentous laxity leads to long term complications like arthritis. Therefore it has to be reconstructed as early as possible if torn.

Anterior Drawer test is diagnostic of a torn ATFL [9] and inversion stress test signifies a torn CFL ${ }^{[10]}$. Anatomic reconstruction of the ligament using Brostrom technique is highly recommended due to advantages of returning the normal joint mechanics and preventing donor site morbidity while preserving the normal gait mechanics ${ }^{[11]}$. However the downside of this procedure being it does not address the subtalar instability that occurs due to CFL rupture. Therefore the non-anatomic reconstruction technique of ChristmanSnook serves a better outcome.

In addition our modification can enhance the lateral stability as two strands of the tendon control the inversion laxity. In this way our technique gives extra importance to the calcaneo-fibular ligament without reducing the strength of peroneus brevis muscle. At the same time we also ensure dynamic stability of the ankle by suturing the ATFL arm of the graft to peroneus brevis so that we recreate the normal gait mechanics.

Hence, this enhancement makes our technique unique and can definitely increase the stability of the ankle joint without donor site morbidity at the peroneus brevis insertion. However, the strength of peroneus brevis could not be confirmed in our study.

In conclusion we found this study to give excellent results in subtalar instability and tibio-talar instability without any donor site morbidity.

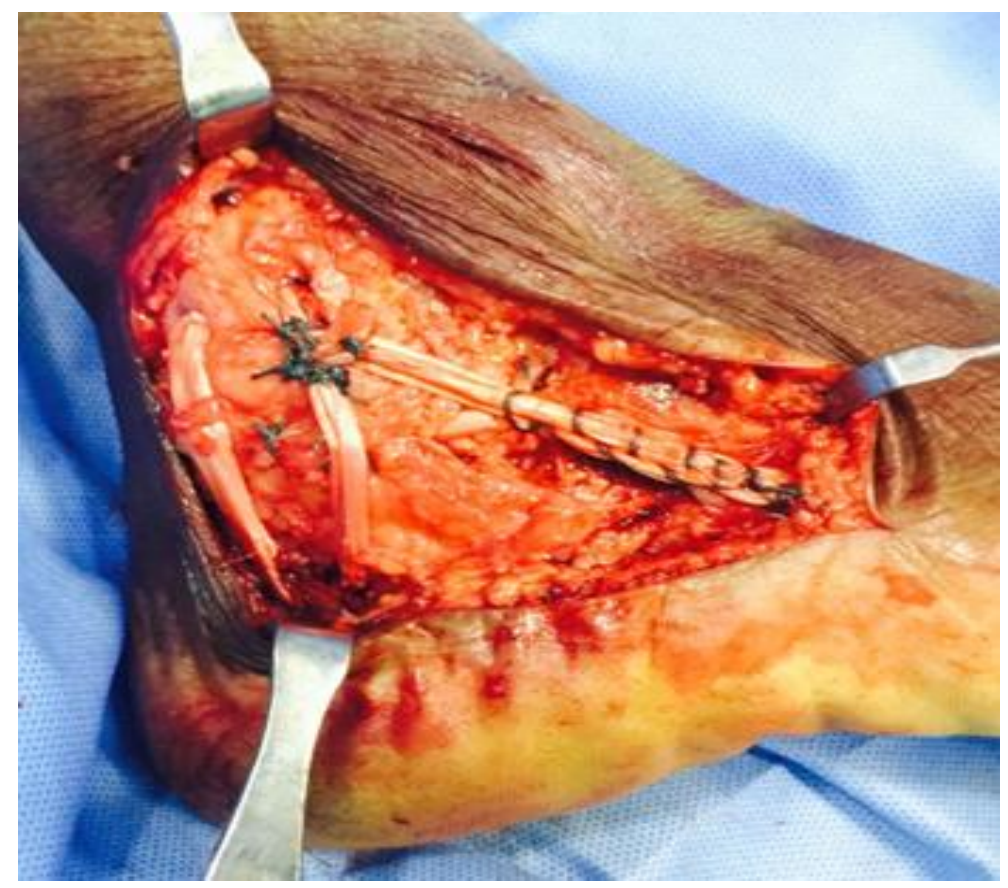

Fig 3: Post reconstruction. 


\section{References}

1. Ellis SJ, Williams BR, Pavlov H, Deland J. Results of Anatomic Lateral Ankle Ligament Reconstruction with Tendon Allograft. HSS Journal. 2011; 7(2):134-140.

2. Brostrom L. Sprained ankles V. treatment and prognosis in recent ligament ruptures. ActaChir Scand. 1966; 5:537-550.

3. Chrisman OD, Snook GA. Reconstruction of lateral ligament tears of the ankle. An experimental study and clinical evaluation of seven patients treated by a new modification of the elmslie procedure. J Bone Joint Surg Am. 1969; 5:904-912.

4. Coughlin MJ, Schenck RC, Jr, Grebing BR, Treme G. Comprehensive reconstruction of the lateral ankle for chronic instability using a free gracilis graft. Foot Ankle Int. 2004; 4:231-241.

5. Lateral instability of the ankle joint. Karlsson J, Lansinger O Clin Orthop Relat Res. 1992; (276):253-61.

6. Sprained ankles. VI. Surgical treatment of "chronic" ligament ruptures. Broström L Acta Chir Scand. 1966; 132(5):551-65.

7. Reconstruction of the lateral ankle ligaments. A biomechanical analysis. Colville MR, Marder RA, Zarins BAm J Sports Med. 1992; 20(5):594-600.

8. Long-term results of the Chrisman-Snook operation for reconstruction of the lateral ligaments of the ankle. Snook GA, Chrisman OD, Wilson TCJ Bone Joint Surg Am. 1985; 67(1):1-7.

9. Lateral ankle stabilization. Modified Lee and ChrismanSnook. Saltrick KR Clin Podiatr Med Surg. 1991 Jul; 8(3):579-600.

10. Lateral instability of the ankle joint. Karlsson J, Lansinger O Clin Orthop Relat Res. 1992; (276):253-61.

11. Surgical Considerations in the Treatment of Ankle Instability. Baumhauer JF, O'Brien TJ Athl Train. 2002; 37(4):458-462. 